\title{
The Novel Modelling Method of Time Series Based on the Internal Model Principle
}

\author{
W. Wang \\ Department of Mathematics, Information School \\ Renmin University of China \\ Beijing, China
}

\begin{abstract}
In this paper, a novel modelling method for time series is considered. Because the influences of the internal or external factors are often inevitable which result in the irregular change of the series, especially in many of the financial time series, the conventional modelling methods, which depend on the autocorrelation of the series, cannot meet the requirements or have a passive response to the factors. To find an effective method which can reflect the intrinsic essence or external influences of the series, the internal model principle, which is used in the synthesis of control systems for revealing the fundamental features of the factors, is introduced. It may open up broad prospects for improving the fitness or prediction ability of the models under the circumstances mentioned above. This paper discusses the possibility and provides some analytical results.
\end{abstract}

Keywords-time series, internal or external factor, modelling, control ideology, internal model principle

\section{INTRODUCTION}

It is well known that a time series is a sequence of observations of a stochastic process, and forecasting the evolution of time series data is important component of operations research because these data often provide the foundation for decision models. Time series analysis provides tools for selecting a model that can be used to forecast of future events. It has played an important role for the evolutionary analysis of series ([1], [2]).

Time series models assume that observations vary according to some probability distribution about an underlying function of time. Usually, people only begin to scratch the surface of the field, restricting their attention to using historical time series data to develop time-dependent models. The methods are appropriate for automatic, short-term forecasting of frequently used information where the underlying causes of time variation are not changing markedly. For stationary time series, an autoregressive moving average model is the basic model. For non-stationary time series, it needs to isolate deterministic trends first, and then model and analyze the surplus remaining stochastic portion ([3]). The limitations and risks existing in traditional methods based on correlation analysis mainly lies in the following three areas: estimated sample autocorrelation is a poor estimator with regular large variance and high relationships with each other; they may produce a completely distorted image of the original series structure; and they are unable to reflect system characteristics accurately ([2], [3]).
In order to make a proper assumption on the model and to make an effective prediction, a modelling method based on dynamic data systems in time series or state space form was developed. The time series is regarded as a realistic way to input response on a stochastic system to uncorrelated white noise ([4], [5]).

At the same time, the continued increase in availability of economic data in recent years and, more importantly, the possibility to construct larger frequency time series, have fostered the use (and development) of statistical and econometric techniques to treat them more accurately ([6]). The paper [7] presents an exposition of structural time series models by which a time series can be decomposed as the sum of a trend, seasonal and irregular components.

However, given those new series it is becoming harder and harder to keep the assumption of a fixed pattern or behavior through time. Indeed, a statistical property of a large proportion of socio-economic series is its evolutive character and, in this context, structural time series models constitute themselves as the appropriate technique, as they allow that each of the typical unobserved components within those series to possess a stochastic nature. In other words, the different components describing the evolution of a time series, trend, seasonality, cycle and irregular, have been traditionally modelled in a deterministic way; when these series are sufficiently large, one is able to assess the volatility of such components and, moreover, it may be reasonable to consider that they evolve randomly over time. That is the starting assumption of structural models.

The forecasting of seasonal economic time series is a challenging problem ([8]). People approach the forecasting challenge from a model-based perspective and adopt the unobserved components time series model. The key feature of those models is the same as the one discussed in [8], that is to say, by using the decomposition of a time series into trend, seasonal, cycle and irregular components. Each component is formulated as a stochastically evolving process over time. The decomposition of an observed time series into unobserved stochastic processes can provide a better understanding of the dynamic characteristics of the series and the way these characteristics change over time. The trend component typically represents the longer term developments of the time series of interest and is often specific as a smooth function of time. The recurring but persistently changing patterns within the years are captured by the seasonal component. In economic 
time series, the cycle component can represent the dynamic features associated with the business cycle (or the output gap) ([9]). In practice, forecasts derived by these methods are likely to be modified by the analyst upon considering information not available from the historical data ([8]). And, by using dynamical innovation or feedback mechanism, or using control method and filter theory, the adaptability of models can be improved ([7], [10]).

Unfortunately, many of the random phenomena, especially in financial time series, the evolutionary processes are often affected by random noise or other unexpected internal or external factors, and they are often changing irregularly. Based on the conventional modelling methods, the models cannot reflect those factors efficiently, let alone to make an effective prediction by them.

In this paper, a novel modelling method for time series is considered. For those series affected by the internal or external factors, the internal model principle is introduced for the problem of modelling. In section 2, a brief summary on the internal model principle is given. In section 3 , the possibility on using internal model principle in time series modelling is discussed, the related time series models are obtained for some cases, and some analytical results are provided. In section 4, some conclusions about the method are presented. It may be full of promise for improving the fitness or prediction ability of the models.

\section{THE BRIEF SUMMARY ON THE INTERNAL MODEL PRINCIPLE}

The internal model principle (IMP) is one of the important synthetic methods for control system. Since its introduced ([11]), it has been still used in the synthesis of control systems ([12]). It concerns the problem of synthesizing regulators processing structural stability with respect to small perturbations in systems parameters. It exploits feedback together with a suitably reduplicated model, internal to the feedback loop, of the dynamic structure of the exogenous reference and disturbance signals which the system was required to process. It is shown that such structure of the regulator is actually necessary ([12]).

The idea of IMP is that the dynamics of external signals should be introduced into the controller for tracking or rejecting, i.e., any control system, which can reject external disturbance or follow reference signal, should contain the dynamics models related with the external signals. The principle also provides the basic idea for representing the internal or external disturbance properly.

On the other hand, from the viewpoint of control theory, the model with the form of the input-output relation can only reflect the components which are not only controllability but also observability, i.e., such a model can not reflect the other factors of the system. So, in order to predict the trends of time series, to find a model employing the other internal or external factors is necessary.

\section{The Method on Modelling for Time Series BASEd ON INTERNAL MODEL PRINCIPLE}

According to the time series theory, the conventional time series models can be translated into the state-space forms ([1], [5]), i.e., such a model has the form with the input of noise and the output of the observable variable. And according to the control theory, such model of times series just reflects the observability parts, as well as the parts of controllability. It cannot reflect the other internal factors that may impact the evolution of the series. So, in order to build a model which is suitable for the varying caused by those factors, we should introduce the dynamic models of those factors in the conventional state-space time series model. The inspiration for such idea can also be summarized just as the situation that, if we want the computer to have the functions of multimedia, we should introduce the mould of multimedia to it. In fact, with the development of information technique, such as big data, and with the increase for the amount of information, we can have more and more knowledge on those factors, which will make the method of using internal model principle possible.

It is based on the internal model principle and the control method that, if the changing of the time series is aroused by certain factors, we should integrate the dynamics of those factors to the model when we want to make a tracking for some signals. In the following, for some cases, we will try to improve the model by using the internal model principle.

For the conventional time series model which can be transformed into the form of state space as follows:

$$
\left\{\begin{array}{l}
\bar{X}(t+1)=A \bar{X}(t)+E d(t) \\
\bar{y}(t)=C \bar{X}(t)
\end{array}\right.
$$

where $\bar{X}(t)$ and $\bar{y}(t)$ are the state variable and observed variable respectively, $d(t)$ is the external disturbance or noise, $A$ and $E$ are the proper matrixes making the equation meaningful.

And we construct an observer for it. For simplicity, we use $X(t)$ as the estimated one of $\bar{X}(t)$ in the observer. So, the observer can be described in following way:

$$
\left\{\begin{array}{l}
X(t+1)=A X(t)+F u(t)+E d(t) \\
y(t)=C X(t)
\end{array}\right.
$$

where $u(t)$ is the function of the error between the estimated one $y(t)$ and the original one $\bar{y}(t)$ of the observed variable, $F$ is the gain of the $u(t)$.

In the following, we will try to introduce some of the internal or external factors to the form of (2) by choosing the proper $e(t)$ such as the following:

$$
u(t)=K_{1} e(t)+v(t)
$$

such that the estimated one $X(t)$ can model the evolution process effectively, even if the variable is suffered from the influence of different kinds of factors. 
A. The Modelling of Time Series When the External and Internal Factors Are Exponential Functions form

For the time series model with the following state-space

$$
\left\{\begin{array}{l}
X(t+1)=A X(t)+F u(t)+E d(t) \\
y(t)=C X(t)
\end{array}\right.
$$

where $X, d$, input $u$, output $y$ are $n, f, m, l$ dimensional vectors, $d(t)$ is the unknown disturbance vector, with the following form:

$$
d(t)=\bar{d} \lambda^{t}+d^{*}
$$

where $\bar{d}=\left(\begin{array}{lll}\bar{d}_{1} & \cdots & \bar{d}_{f}\end{array}\right)^{\tau}, d^{*}=\left(\begin{array}{lll}d_{1}^{*} & \cdots & d_{f}^{*}\end{array}\right)^{\tau}$ are the constant vectors but the values are unknown, $\lambda$ is the known constant.

We intend to find a model for $y(t)$ such that it can approach the real characteristics or external factors $y_{r}(t)$ as closely as possible, here $y_{r}(t)$ is also a vector with dimensional of $l$. i.e.

$$
\lim _{t \rightarrow \infty} y(t)=\bar{y}_{r} \lambda^{t}+y_{r}^{*}
$$

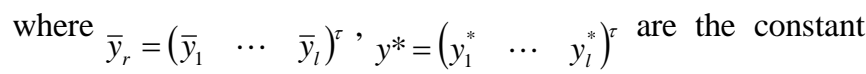
vectors but the values are unknown, $A^{\tau}$ means the transpose of A.

Let the error signal is as follows

$$
e(t)=y(t)-\bar{y}_{r} \lambda^{t}-y_{r}^{*}
$$

In order to $e(t)=0$, we introduce the quantity $q_{1}(t)$, such that

$$
q_{1}(t+1)=q_{1}(t)+e(t)
$$

According to the internal model principle, the control input should contain the term of $\lambda^{t}$. In order to reflect the exponential increasing of index we usually have the internal dynamic system, so we introduce the following dynamical system

$$
q_{2}(t+1)=\lambda q_{2}(t)+q_{1}(t)
$$

From (8) we know that, because of $e(t)$ contains the term of $\lambda^{t}$, and the characteristic roots of (8) is $\lambda$, so $q_{2}$ can reflect the change of $\lambda^{t}$, so we can extend (4) into the following form

$$
\begin{aligned}
\left(\begin{array}{l}
X(t+1) \\
q_{1}(t+1) \\
q_{2}(t+1)
\end{array}\right)= & \left(\begin{array}{ccc}
A & 0 & 0 \\
C & I_{l} & 0 \\
0 & I_{l} & \lambda I_{l}
\end{array}\right)\left(\begin{array}{l}
X(t) \\
q_{1}(t) \\
q_{2}(t)
\end{array}\right)+\left(\begin{array}{l}
F \\
0 \\
0
\end{array}\right) u(t)+\left(\begin{array}{l}
E \\
0 \\
0
\end{array}\right)\left(\bar{d} \lambda^{t}+d^{*}\right)-\left(\begin{array}{l}
0 \\
I \\
0
\end{array}\right)\left(\left(\bar{y}_{l} \lambda^{t}+y_{l}^{*}\right) B y\right. \\
& \text { introducing the feedback as follows: } \\
u(t)= & K_{1} X(t)+K_{2} q_{1}(t)+K_{3} q_{2}(t)
\end{aligned}
$$

We can obtain the closed-loop form of the extend system as follows:

$$
\left(\begin{array}{c}
X(t+1) \\
q_{1}(t+1) \\
q_{2}(t+1)
\end{array}\right)=\left(\begin{array}{ccc}
A+F K_{1} & F K_{2} & F K_{3} \\
C & I_{l} & 0 \\
0 & I_{l} & \lambda I_{I}
\end{array}\right)\left(\begin{array}{c}
X(t) \\
q_{1}(t) \\
q_{2}(t)
\end{array}\right)+\left(\begin{array}{l}
E \\
0 \\
0
\end{array}\right)\left(\bar{d} \lambda^{t}+d^{*}\right)-\left(\begin{array}{l}
0 \\
I \\
0
\end{array}\right)\left(\overline{y_{l}} \lambda^{t}+y_{l}^{*}\right)
$$

Then based on the results on the stability of discrete time systems, we can choose all characteristic roots of the system in the unit circle, then we can have the following results:

$$
\begin{aligned}
& \lim _{t \rightarrow \infty} X(t)=\bar{x} e^{\lambda t}+x^{*} \\
& \lim _{t \rightarrow \infty} q_{1}(t)=\bar{q}_{1} \lambda^{t}+q_{1}^{*} \\
& \lim _{t \rightarrow \infty} q_{2}(t)=\bar{q}_{2} \lambda^{t}+q_{2}^{*}
\end{aligned}
$$

where $\bar{x}, x^{*}, \bar{q}_{1}, q_{1}^{*}, \bar{q}_{2}, q_{2}^{*}$ are all the constant vectors. The result indicates that the variable $X(t)$ can model the exponential functions asymptotically.

\section{B. The Modelling of Time Series When the External and Internal Factors Are Periodical Functions}

As to the model for the variables which governed by the system of (4) with the external and internal factors, which are all in the form of periodical functions, we can introduce internal model in following way

$$
\left\{\begin{array}{l}
q_{1}(t+1)=q_{1}(t)+y(t)-\alpha_{1} \sin \beta t-\alpha_{2} \cos \beta t-\alpha_{3} \\
q_{2}(t+1)=2 \cos \beta \times q_{2}(t)+q_{1}(t)-q_{3}(t) \\
q_{3}(t+1)=q_{2}(t)
\end{array}\right.
$$

And the extend system of (4) can be obtained as follows:

$$
\left(\begin{array}{l}
X(t+1) \\
q_{1}(t+1) \\
q_{2}(t+1) \\
q_{3}(t+1)
\end{array}\right)=\left(\begin{array}{cccc}
A & 0 & 0 & 0 \\
C & I_{l} & 0 & 0 \\
0 & I_{l} & 2 \cos \beta \times I_{l} & -I_{l} \\
0 & 0 & I_{l} & 0
\end{array}\right)\left(\begin{array}{c}
X(t) \\
q_{1}(t) \\
q_{2}(t) \\
q_{3}(t)
\end{array}\right)+\left(\begin{array}{c}
F u(t)+E d(t) \\
-I_{l}\left(\alpha_{1} \sin \beta t+\alpha_{2} \cos \beta t+\alpha_{3}\right) \\
0 \\
0
\end{array}\right)
$$

By introducing the feedback of the following

$$
u(t)=K_{1} X(t)+K_{2} q_{1}(t)+K_{3} q_{2}(t)+K_{4} q_{3}(t)
$$

the state matrix of the close-loop system is as follows:

$$
\left(\begin{array}{cccc}
A+F K_{1} & F K_{2} & F K_{3} & F K_{4} \\
C & I_{l} & 0 & 0 \\
0 & I_{l} & 2 \cos \beta \times I_{l} & -I_{l} \\
0 & 0 & I_{l} & 0
\end{array}\right)
$$

Based on the results on the stability of discrete time systems, we can choose all the characteristic roots of the system in the unit circle, then we can have the following results:

$$
\begin{gathered}
\lim _{t \rightarrow \infty} X(t)=x_{11} \sin \beta t+x_{12} \cos \beta t+x_{13} \\
\lim _{t \rightarrow \infty} q_{1}(t)=q_{11} \sin \beta t+q_{12} \cos \beta t+q_{13} \\
\lim _{t \rightarrow \infty} q_{2}(t)=q_{21} \sin \beta t+q_{22} \cos \beta t+q_{23} \\
\lim _{t \rightarrow \infty} q_{3}(t)=q_{31} \sin \beta t+q_{32} \cos \beta t+q_{33}
\end{gathered}
$$

From (13) we know that, as $t \rightarrow \infty$, according to the relationship of $q_{1}(t), q_{2}(t), q_{3}(t)$ we can obtain that $q_{1}(t)=0$, i.e. $e(t) \rightarrow 0$. That is to say, the output can follow the required form of periodical function. The results indicate 
that the variable $X(t)$ can model the characteristics of periodical functions.

\section{The Modelling of Time Series When the External and Internal Factors Are the Combination of Periodical Functions and Exponential Functions}

As to the model for the variables which governed by the system (4) with the external and internal factors, which are all the combination of the periodical functions and exponential functions, we can introduce internal model as the following form:

$$
\left\{\begin{array}{l}
q_{1}(t+1)=q_{1}(t)+e(t) \\
q_{2}(t+1)=\lambda q_{2}(t)+q_{1}(t) \\
q_{3}(t+1)=2 \cos \beta \times q_{2}(t)+q_{1}(t)-q_{3}(t) \\
q_{4}(t+1)=q_{3}(t)
\end{array}\right.
$$

where

$$
e(t)=y(t)-\bar{y}_{r} \lambda^{t}-\alpha_{1} \sin \beta t-\alpha_{2} \cos \beta t-y_{r}^{*} .
$$

So, the extend system of (4) is as follows:

$$
\begin{gathered}
\left(\begin{array}{c}
X(t+1) \\
q_{1}(t+1) \\
q_{2}(t+1) \\
q_{3}(t+1) \\
q_{4}(t+1)
\end{array}\right)=\left(\begin{array}{ccccc}
A & 0 & 0 & 0 & 0 \\
C & I_{l} & 0 & 0 & 0 \\
0 & I_{l} & \lambda I_{l} & 0 & 0 \\
0 & 0 & I_{l} & 2 \cos \beta \times I_{l} & -I_{l} \\
0 & 0 & 0 & I_{l} & 0
\end{array}\right)\left(\begin{array}{c}
X(t) \\
q_{1}(t) \\
q_{2}(t) \\
q_{3}(t) \\
q_{4}(t)
\end{array}\right)+ \\
\left.\begin{array}{c}
F u(t)+E d(t) \\
-I_{l}\left(\bar{y}_{r} \lambda^{t}+\alpha_{1} \sin \beta t+\alpha_{2} \cos \beta t+y_{r}^{*}\right) \\
0 \\
0
\end{array}\right)
\end{gathered}
$$

By introducing the following form of feedback:

$$
u(t)=K_{1} X(t)+K_{2} q_{1}(t)+K_{3} q_{2}(t)+K_{4} q_{3}(t)+K_{5} q_{4}(t)
$$

we obtain the state matrix of the close-loop system as follows:

$$
\left(\begin{array}{ccccc}
A+F K_{1} & F K_{2} & F K_{3} & F K_{4} & F K_{5} \\
C & I_{l} & 0 & 0 & 0 \\
0 & I_{l} & \lambda I_{l} & 0 & 0 \\
0 & 0 & I_{l} & 2 \cos \beta \times I_{l} & -I_{l} \\
0 & 0 & 0 & I_{l} & 0
\end{array}\right)
$$

Then based on the results on the stability of discrete time systems, we can choose all the characteristic roots of the system in the unit circle, then we can have the following results:

$$
\begin{aligned}
& \lim _{t \rightarrow \infty} X(t)=x_{11} \lambda^{t}+x_{12} \sin \beta t+x_{13} \cos \beta t+x_{14} \\
& \lim _{t \rightarrow \infty} q_{1}(t)=q_{11} \lambda^{t}+q_{12} \sin \beta t+q_{13} \cos \beta t+q_{14} \\
& \lim _{t \rightarrow \infty} q_{2}(t)=q_{21} \lambda^{t}+q_{22} \sin \beta t+q_{23} \cos \beta t+q_{24} \\
& \lim _{t \rightarrow \infty} q_{3}(t)=q_{31} \lambda^{t}+q_{32} \sin \beta t+q_{33} \cos \beta t+q_{34} \\
& \lim _{t \rightarrow \infty} q_{4}(t)=q_{41} \lambda^{t}+q_{42} \sin \beta t+q_{43} \cos \beta t+q_{44}
\end{aligned}
$$

From above results we know that, as $t \rightarrow \infty$, according to the relationship of $q_{1}(t), q_{2}(t), q_{3}(t)$ we can obtain the conclusion that $q_{1}(t)=0$, i.e. $e(t) \rightarrow 0$. That is to say, the output $X(t)$ can follow the required form.

The result indicates that the variable $X(t)$ can model the characteristics of periodical functions and exponential functions. By now, we have the theoretical results on time series modelling for three cases above. They are the results that may open up broad possible for building the model effectively. As to other more complex cases, they need further considerations.

\section{CONCLUSIONS}

In this paper, a novel modelling method for time series is considered for the cases that the time series are influenced by some of the internal or external factors, which result in the irregular change of the series. The conventional methods, which depend on the autocorrelation of the series, cannot meet the requirements or have a passive reflection to the factors. Based on the internal model principle, the method on improving the adaptability of the model is proposed. This paper discusses the possibility, and some analytical results are obtained. It may be full of promise for improving the fitness and the ability of prediction of the models under the circumstances mentioned above. Of course, there are many more complex cases need further considerations.

\section{REFERENCES}

[1] Box, G.E.P, Jenkins, G.M. \& Reinsel, G.C., Time Series Analysis: Forecasting, and Control, 4th Edition, John Wiley \& Sons, Inc., 2008.

[2] Chatfield, C., The Analysis of Time Series: An Introduction, Sixth Edition, Chapman \& Hall/CRC Texts in Statistical Science, 2003.

[3] Shumway, R.H. \& Stoffer, D.S., Time Series Analysis and Its Applications, Springer-Verlag, New York, 2000.

[4] West, M. \& Harrison, J., Baysian Forecasting and Dynamic Models, Second Edition, Springer-Verlag, New York, 1997.

[5] Durbin, J. \& Koopman, S. J., Time Series Analysis by State Space Methods. Oxford: Oxford University Press. 2001.

[6] Gaynor, P.E. \& Kirkpatrick, R.C., Introduction to Time-Series Modeling and Forecasting in Business and Economics, McGraw-Hill, New York, 1994.

[7] Jalles, J.T., Structural time series models and the Kalman filter: a concise review, FEUNL Working Paper, No. 541, June 19, 2009.

[8] Ledolter, J. \& Bisgaard, S., Challenges in constructing time series models from process data, Quality and Reliability Engineering International, 27, pp. 165--178, 2011.

[9] Groen, J.J.J., Paap, R. \& Ravazzolo, F., Real-Time Inflation Forecasting in a Changing World, Federal Reserve Bank of New York Staff Reports, No. 388, August 2009; revised May 2012.

[10] Wang, W., The method on improving the adaptability of time series models based on dynamical innovation, Communication in Computer and Informa-tion Science Series, 288, pp.210-217, 2012.

[11] Francis, B.A. \& Wonham, W.M., The internal model principle of control theory, Automatica, 12(5), pp. 457-465, 1976.

[12] Wieland, P., Sepulchre, R. \& Allgöwer, F., An internal model principle is necessary and sufficient for linear output synchronization, Automatica, 47, pp.1068-1074, 2011. 